\title{
Fungal Internal Carotid Artery Aneurysm Treated by Trapping and High-Flow Bypass: A Case Report and Literature Review
}

\author{
Junya YAmAgUCHI, ${ }^{1}$ Teppei KAWABATA, ${ }^{1}$ Ayako MOTOMURA, ${ }^{2}$ \\ Norikazu HATANO, ${ }^{1}$ and Yukio SEKI ${ }^{1}$ \\ ${ }^{1}$ Department of Neurosurgery, Nagoya Daini Red Cross Hospital, Nagoya, Aichi \\ ${ }^{2}$ Department of Neurosurgery, Daido Hospital, Nagoya, Aichi
}

\begin{abstract}
We report a case of unruptured fungal internal carotid artery (ICA) aneurysm and review the pertinent literature. A 79-year-old man presented with decreased visual acuity on the right side, and he was diagnosed with retrobulbar optic neuritis. Medical treatment with steroids resulted in Aspergillus meningoencephalitis spreading to the bottom of bilateral frontal lobes, caused by an intracranial extension of sphenoid sinusitis. Magnetic resonance imaging (MRI) performed 26 days after the start of antifungal therapy showed a denovo right ICA aneurysm projecting anteriorly into the sphenoid sinus. As the aneurysm grew rapidly, it was trapped surgically after establishing a high-flow bypass from the external carotid artery to the middle cerebral artery. The patient's postoperative course was uneventful. Anti-fungal medication was continued until plasma concentrations of beta-D-glucan decreased to within normal limits. Although fungal ICA aneurysm carries a high mortality rate, early detection and prompt treatment by trapping and high-flow bypass can lead to good clinical outcome.
\end{abstract}

Key words: fungal aneurysm, Aspergillus, trapping, high-flow bypass

\section{Introduction}

It has been reported that fungal internal carotid artery (ICA) aneurysm is very rare, and its mortality rate is very high. ${ }^{1)}$ Owing to the increase in the number of immunocompromised patients resulting from the widespread use of steroidal, chemotherapy, and immunosuppressive agents, the incidence of fungal ICA aneurysm is expected to increase. ${ }^{2)}$ Recently, there have been several case reports of fungal ICA aneurysms in which performance of endovascular surgery tended to be associated with increased survival rates. ${ }^{1,3-6)}$ Here we report a case of unruptured fungal ICA aneurysm that was treated successfully by surgical trapping and high-flow bypass and present a review of the pertinent literature.

\section{Case Description}

A 79-year-old man with a past history of diabetes mellitus and tuberculosis presented with decreased visual acuity on the right side and was diagnosed with retrobulbar optic neuritis. Medical treatment with a steroid (prednisolone

Received August 5, 2015; Accepted October 26, 2015
$40 \mathrm{mg} /$ day) was initiated and continued for 2 months but provided no symptom relief and subsequently resulted in a recurrence of tuberculosis. The steroid was discontinued, antituberculosis therapy was initiated. As the patient's cognitive function began to decline 2 weeks later, cerebrospinal fluid (CSF) examination and MRI were performed. A CSF examination showed an elevated cell count and the protein level (cell count: 139/dL, glucose: $72 \mathrm{mg} / \mathrm{dL}$, protein: 101 $\mathrm{mg} / \mathrm{dL}$ ) and was positive for the Aspergillus antigen. Fluid attenuated inversion recovery (FLAIR) imaging revealed an isointense lesion in the right side of the sphenoid sinus and a high-intensity lesion spreading at the bottom of the bilateral frontal lobes (Fig. 1A, B). Based on these findings, a diagnosis of fungal meningoencephalitis due to intracranial extension of Aspergillus sinusitis was made.

Antifungal therapy with amphotericin B was started, but it was subsequently changed to micafungin because of an adverse effect on renal function. Follow-up CSF examination and MRI performed 26 days after starting of antifungal therapy showed decreased CSF cell counts (cell count: 6/dL, glucose: $71 \mathrm{mg} / \mathrm{dL}$, protein: $130 \mathrm{mg} / \mathrm{dL}$ ), some shrinkage of the sphenoid sinus lesion, and a de novo right ICA aneurysm projecting anteriorly into the sphenoid sinus at the C3 portion (Fig. 1C). It was noted that 

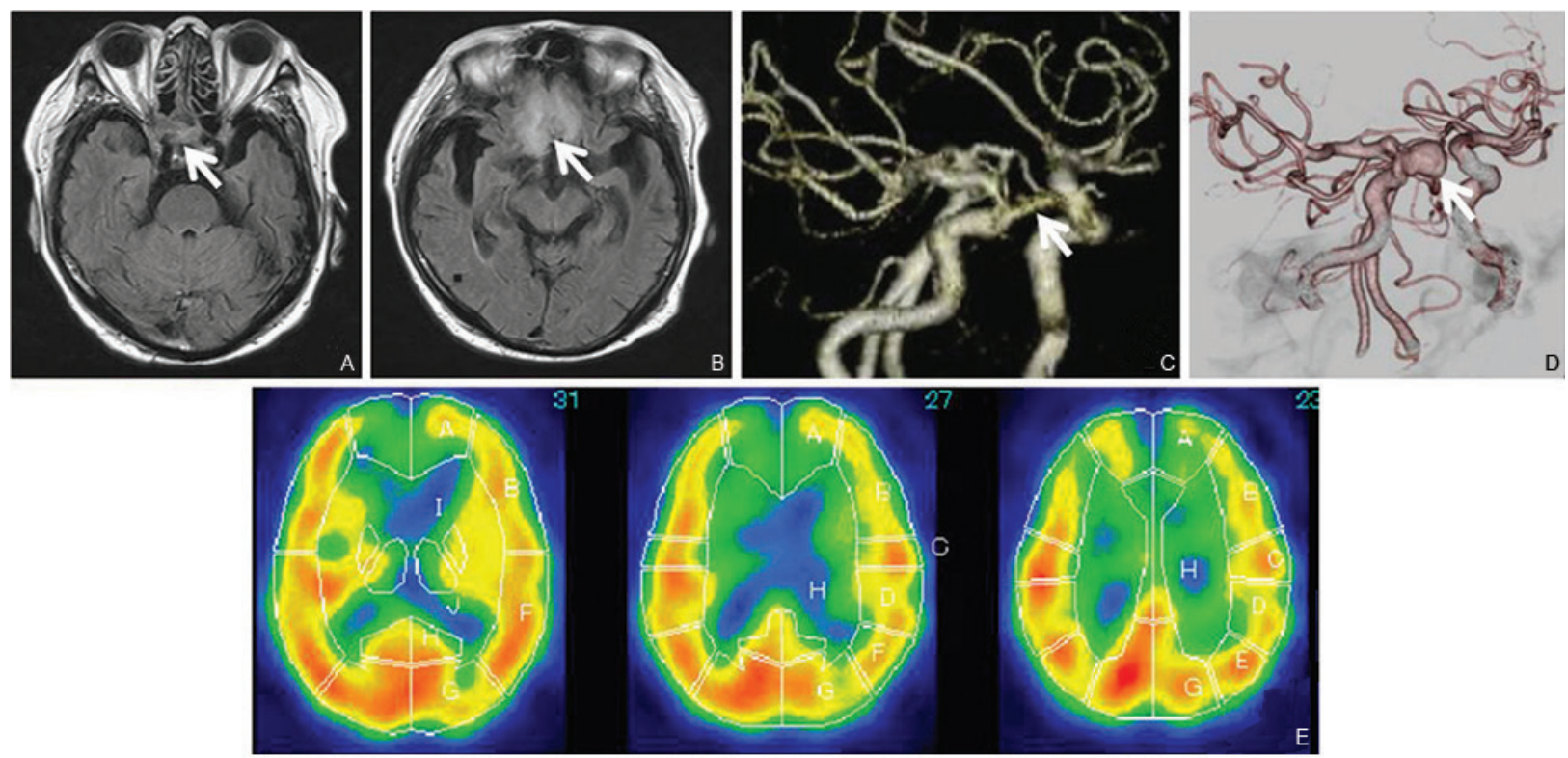

Fig. 1 A: Axial FLAIR image showing an isointense lesion (arrow) in the right side of the sphenoid sinus extending to the orbital apex and the cavernous portion of the ICA. B: Axial FLAIR image showing a high-intensity lesion (arrow) at the base of the bilateral frontal lobes, suggesting meningoencephalitis due to intracranial extension of sphenoid sinusitis. C: MRA image, right anterior oblique view, showing an aneurysm (arrow) projecting anteriorly at the C3 portion of the right ICA. D: 3D-CT angiography, right anterior oblique view, showing rapid growth of the aneurysm (arrow). Note that bilateral anterior cerebral arteries distal to the anterior communicating artery are not opacified, suggesting the presence of a stenosis caused by frontal base meningoencephalitis. E: Pre-operative single photon emission computed tomography showing a hypoperfusion area in the anterior cerebral artery distribution. FLAIR: fluid attenuated inversion recovery, ICA: internal carotid artery, MRA: magnetic resonance angiogram, 3D-CT: three-dimensional computed tomography.

bilateral anterior cerebral arteries (ACAs) distal to the anterior communicating artery (AcoA) were pooly visualized, suggesting the presence of arterial stenosis caused by frontal base meningoencephalitis. Because a threedimensional computed tomography (3D-CT) angiogram taken 5 days later showed rapid growth of this fungal aneurysm (from $9 \mathrm{~mm} \times 3 \mathrm{~mm}$ to $11 \mathrm{~mm} \times 10 \mathrm{~mm}$ ) (Fig. 1D), we planned an emergency operation to prevent the aneurysm rupturing, which might have resulted in fatal epistaxis. Pre-operative single photon emission computed tomogram (SPECT) showed presence of a hypoperfusion area in the anterior cerebral artery distribution (Fig. 1E).

Under endotracheal general anesthesia, a high-flow bypass from the external carotid artery to the M2 portion of the middle cerebral artery (MCA) was established on the right side using a radial artery graft following a superficial temporal artery (STA) - MCA assist bypass to the M4 portion distal to the anastomosis site of the high-flow bypass. Subsequently, the right ICA was trapped between its origin in the neck and the $\mathrm{C} 2$ portion of the ICA. A marked granulomatous change was seen in the dura mater in the frontal base. The right ophthalmic artery was involved in the trapping, as the patient's right visual acuity consisting of only light perception just before the operation. We believed that performing anterior clinoidectomy to expose the ophthalmic artery might further accelerate the intracranial invasion of Aspergillus species. Finally, we anastomosed the frontal branch of the STA to the cortical branch of the right ACA to increase perfusion in its territory.

The patient's postoperative course was uneventful. MRI taken 4 days after the operation showed patency of both the radial artery graft and the frontal branch of the right STA (Fig. 2A). There was no new cerebral infarction. The right ICA had been obliterated and the aneurysm was not visualized. Post-operative SPECT taken 2 weeks after the operation showed improved perfusion in the right anterior cerebral artery distribution (Fig. 2B). Antifungal treatment was continued with itraconazole until plasma concentrations of beta-D-glucan had decreased to within normal limits. Fifty-five days after the operation, the patient was transferred to a rehabilitation hospital because of persisting cognitive function impairment.

\section{Discussion}

Intracranial fungal infection is a rare condition, accounting for $0.8 \%$ of all intracranial infections. ${ }^{7)}$ There are three routes through which a fungus invades the intracranial space. ${ }^{8)}$ The first route is hematogenous dissemination 

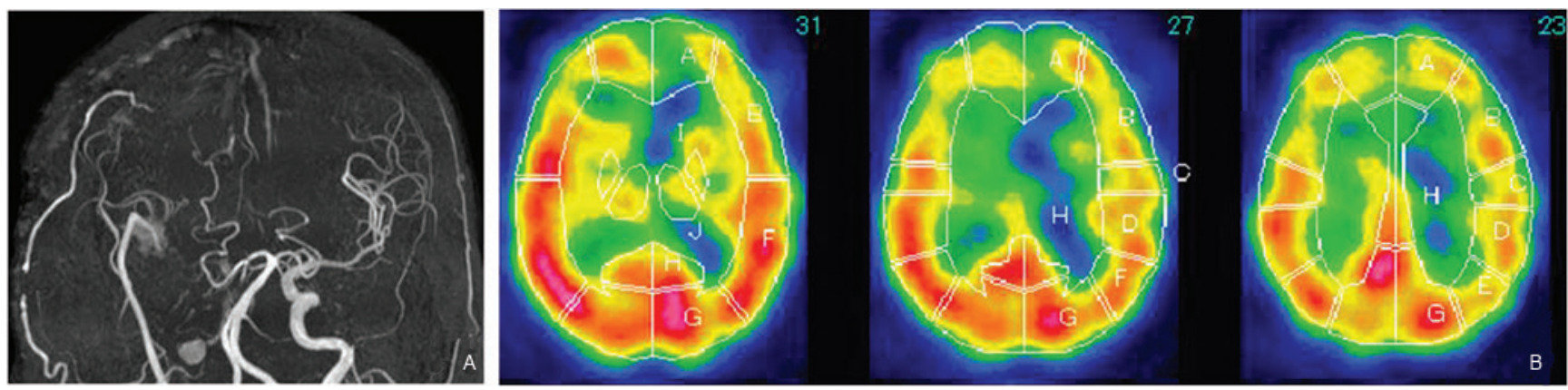

Fig. 2 A: MRI image taken 4 days after the operation showing patency of both the radial artery graft and the frontal branch of the right superficial temporal artery. The right ICA has been obliterated, and the aneurysm is not visualized. B: Post-operative single photon emission computed tomogram showing improved perfusion in the right anterior cerebral artery distribution. ICA: internal carotid artery, MRI: magnetic resonance imaging.

from a remote extracranial site, such as the lung. The second route is through direct invasion from a contiguous cranial focus, as in fungal paranasal sinusitis. The third route is through contamination caused by improper neurosurgical procedures. In cases of hematogenous dissemination, fungal aneurysms tend to develop at distal sites of the ACA, MCA, or posterior cerebral artery (PCA) rather than the ICA, as is the case for mycotic aneurysms that result from infectious endocarditis. ${ }^{8)}$ In contrast, when the fungus invades from the paranasal sinus, intracavernous and supraclinoid portions of the ICA are frequently affected like the present case. Owing to the recent increase in the number of immunocompromised patients, it is expected that the incidence of fungal ICA aneurysms will likewise increase, just as the number of patients with fungal paranasal sinusitis is increasing. ${ }^{2)}$ However, it is often difficult to diagnose a fungal aneurysm before it ruptures because its presentation is insidious (e.g., the fungal sinusitis presents with vague symptoms, such as headache, rhinorrhea, disorder of the sense of smell, or facial discomfort). ${ }^{6)}$ Among 18 cases of fungal ICA aneurysms described below, just 5 patients were diagnosed before its rupture. Thus, it is important to have a high index of suspicion of a fungal ICA aneurysm in patients with fungal paranasal sinusitis, especially when orbital apex syndrome is present. ${ }^{8)}$ In the present case, the patient's cognitive function impairment triggered a thorough study of the brain, including MRI, and therefore, a rapidly developing ICA aneurysm could be detected before its rupture.

Aspergillus is the most common cause of fungal aneurysms. This is because Aspergillus is most frequently found in deep mycosis and has the characteristic of vasocentric tropism. The key molecule that leads to aneurysm formation is the elastase produced by Aspergillus. Elastase decomposes elastine, a major component of the vascular wall, and induces inflammation in all layers of the vascular wall.9)
By searching the English-language medical literature, we were able to collect 18 case reports of fungal ICA aneurysms (Table 1). Among these 18 cases, 11 of the patients had died. Of 9 cases in which surgical or endovascular intervention was performed, 7 patients survived. Among the 9 cases, endovascular surgery was selected in 6 cases and open surgery in 3 cases. In contrast, in the 9 cases in which aneurysms were treated conservatively, all the patients died. These data demonstrate that early intervention is warranted to obliterate fungal ICA aneurysms and prevent patient deaths. In endovascular surgery for fungal ICA aneurysms, parent artery occlusion with a detachable coil or balloon was the main procedure selected. It is less invasive and has merit to provide immediate hemostasis against a background of ongoing epistaxis. Recently Kim et al. reported a case of a patient who presented with severe epistaxis due to rupture of a fungal cavernous ICA aneurysm. The aneurysm was successfully treated with a graft stent. However, two aneurysms developed later, downstream of the affected ICA, and the authors suspected that these aneurysms were mycotic and had developed owing to a graft stent infection. ${ }^{6)}$ As no such a complication was reported in other patients who received endovascular parent artery occlusion, preservation of blood flow through a bioprosthesis in the setting of an active infection may result in colonization with the circulating fungus. Hurst et al. reported a case in which a fungal ICA aneurysm extended intradurally and caused a fatal subarachnoid hemorrhage after parent artery occulusion with a coil. ${ }^{3)}$ These reports suggest that treating fungal ICA aneurysms with foreign bodies, such as a stent or coil, might not be a promising approach.

In the context of surgical cases, Bowers et al. reported a case of a patient with ruptured fusiform paraclinoid ICA aneurysm caused by Aspergillus invasion. Although clip-wrapping of the aneurysm was performed, the patient died owing to occlusion of the affected ICA and a complete infarct in its distribution. ${ }^{10)}$ Loeys et al. reported a case 


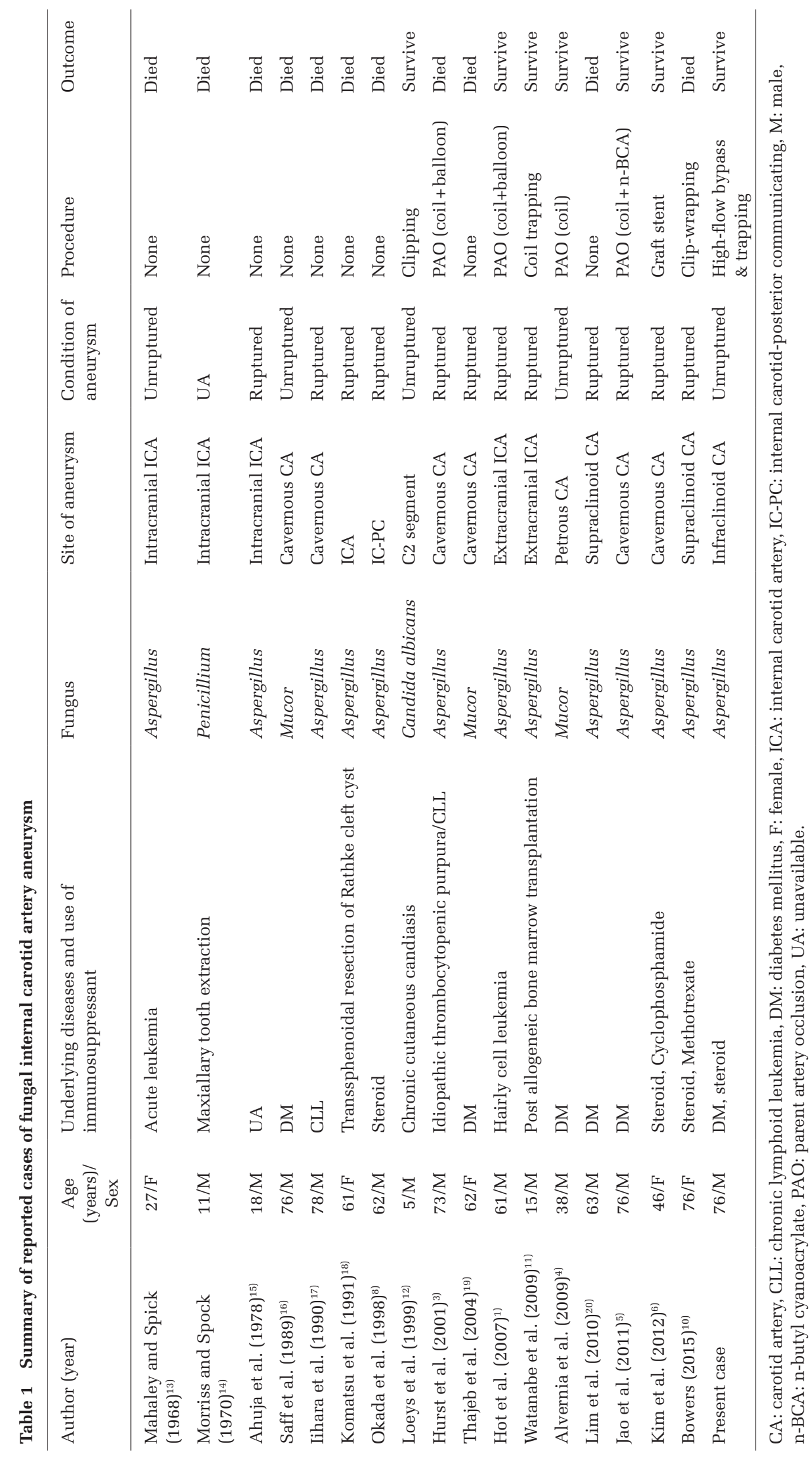


of fungal ICA aneurysm in a child with familial chronic mucocutaneous candidiasis. In this patient, clipping the aneurysm after 3 months of antimycotic treatment led to a favorable outcome. ${ }^{12)}$ Several issues need to be considered when planning surgery for fungal ICA aneurysms. First, fungal aneurysms are prone to rupture and therefore require prompt obliteration. Second, surgical manipulation of the skull base, such as anterior clinoidectomy, carries the potential risk of disseminating the fungus to the central nervous system. Third, considering the pathological changes to the affected arterial wall, neck clipping the aneurysm in the acute stage may not be reliable. In the present case, we performed trapping of the ICA aneurysm with an extracranial-intracranial high-flow bypass. This procedure precluded the risk of rupture, thereby avoiding ischemia and procedure-related dissemination of the fungal infection. There may be an arguement about the necessity of trapping the aneurysm. Another option of surgical procedure is affected ICA ligation at the neck with a high-flow bypass. However, in this setting the risk of rupture could not be precluded until the aneurysm becomes completely thrombosed and is isolated from the established collateral circulation. As for the high-flow bypass in this case, we believed it was mandatory because 3D-CT angiogram did not show the AcoA and bilateral distal ACAs. Probably collateral flow through the leptomeningeal anastomosis was supplying their territories. In such a setting, a low-flow bypass may not be enough to avoid ischemia in the ICA territory after its occlusion.

In conclusion, in cases of invasive paranasal sinus aspergillosis, early detection of a fungal ICA aneurysm is paramount. Prompt trapping of the aneurysm with an appropriate bypass combined with antifungal medications is a reliable treatment regimen for particular group of patients.

\section{Conflicts of Interest Disclosure}

The authors have no personal, financial, or institutional interest in any of the drugs, materials, or devices presented in this article. All authors who are members of The Japan Neurosurgical Society (JNS) have registered online Selfreported COI Disclosure Statement Forms through the website for JNS members.

\section{References}

1) Hot A, Mazighi M, Lecuit M, Poirée S, Viard JP, Loulergue P, Suarez F, Dupont B, Merland JJ, L ortholary O: Fungal internal carotid artery aneurysms: successful embolization of an Aspergillus-associated case and review. Clin Infect Dis 45: e156-e161, 2007

2) Arnold TM, Sears CR, Hage CA: Invasive fungal infections in the era of biologics. Clin Chest Med 30: 279-286, vi, 2009
3) Hurst RW, Judkins A, Bolger W, Chu A, Loevner LA: Mycotic aneurysm and cerebral infarction resulting from fungal sinusitis: imaging and pathologic correlation. AJNR Am J Neuroradiol 22: 858-863, 2001

4) Alvernia JE, Patel RN, Cai DZ, Dang N, Anderson DW, Melgar M: A successful combined endovascular and surgical treatment of a cranial base mucormycosis with an associated internal carotid artery pseudoaneurysm. Neurosurgery 65: 733-740; discussion 740, 2009

5) Jao SY, Weng HH, Wong HF, Wang WH, Tsai YH: Successful endovascular treatment of intractable epistaxis due to ruptured internal carotid artery pseudoaneurysm secondary to invasive fungal sinusitis. Head Neck 33: 437-440, 2011

6) Kim YC, Lee H, Ryu HH, Beom SH, Yang Y, Kim S, Chin HJ: Aspergillus-associated cerebral aneurysm successfully treated by endovascular and surgical intervention with voriconazole in lupus nephritis patient. J Korean Med Sci 27: 317-320, 2012

7) Kamei S, Takasu T: Nationwide survey of the annual prevalence of viral and other neurological infections in Japanese inpatients. Intern Med 39: 894-900, 2000

8) Okada Y, Shima T, Nishida M, Yamane K, Yoshida A: Subarachnoid hemorrhage caused by Aspergillus aneurysm as a complication of transcranial biopsy of an orbital apex lesion-case report. Neurol Med Chir (Tokyo) 38: 432-437, 1998

9) Kothary MH, Chase T, Macmillan JD: Correlation of elastase production by some strains of Aspergillus fumigatus with ability to cause pulmonary invasive aspergillosis in mice. Infect Immun 43: 320-325, 1984

10) Bowers CA, Saad D, Clegg DO, Ng P, Clayton F, Haydoura S, Schmidt RH: Rapidly fatal internal carotid artery mycotic aneurysm rupture in a rheumatoid patient taking a tnf- $\alpha$ inhibitor: case report and literature review. J Neurol Surg A Cent Eur Neurosurg 76: 249-254, 2015

11) Watanabe $T$, Okada $T$, Okada $C$, Onishi $T$, Watanabe $H$, Okamoto Y, Kitamura Y, Manabe S, Matsubara S, Kageji T, Iwai A: An aspergillotic aneurysm of the internal carotid artery following allogeneic bone marrow transplantation: successful management with catheter coil embolization and long-term antifungal agents. Transpl Infect Dis 11: 49-53, 2009

12) Loeys BL, Van Coster RN, Defreyne LR, Leroy JG: Fungal intracranial aneurysm in a child with familial chronic mucocutaneous candidiasis. Eur J Pediatr 158: 650-652, 1999

13) Mahaley M, Spick A: An unusual case of intracranial aneurysm, in Smith J (ed): Neuro-Ophthalmology. St. Louis, Mosby, 1968, pp 158-166

14) Morriss FH, Spock A: Intracranial aneurysm secondary to mycotic orbital and sinus infection. Report of a case implicating penicillium as an opportunistic fungus. Am J Dis Child 119: 357-362, 1970

15) Ahuja GK, Jain N, Vijayaraghavan M, Roy S: Cerebral mycotic aneurysm of fungal origin. Case report. J Neurosurg 49: 107-110, 1978

16) Saff G, Frau M, Murtagh FR, Silbiger ML: Mucormycosis associated with carotid cavernous fistula and cavernous carotid mycotic aneurysm. J Fla Med Assoc 76: 863-865, 1989 
17) Iihara K, Makita $Y$, Nabeshima S, Tei T, Keyaki A, Nioka $\mathrm{H}$ : Aspergillosis of the central nervous system causing subarachnoid hemorrhage from mycotic aneurysm of the basilar artery-case report. Neurol Med Chir (Tokyo) 30: 618-623, 1990

18) Komatsu Y, Narushima K, Kobayashi E, Tomono Y, Nose T: Aspergillus mycotic aneurysm-case report. Neurol Med Chir (Tokyo) 31: 346-350, 1991

19) Thajeb P, Thajeb T, Dai D: Fatal strokes in patients with rhino-orbito-cerebral mucormycosis and associated vasculopathy. Scand J Infect Dis 36: 643-648, 2004
20) Lim SC, Choi JU, Bae SH: Rapid development of an infectious aneurysm of the internal carotid artery from orbital apex syndrome. Otolaryngol Head Neck Surg 142: 294-295, 2010

Address reprint requests to: Junya Yamaguchi, MD, Department of Neurosurgery, Nagoya Daini Red Cross Hospital, 2-9 Myouken, Showa-Ku, Nagoya 466-8650, Japan. e-mail: jyamaguchi@nagoya2.jrc.or.jp 